\title{
Synthesis and in Vitro Cytotoxic Evaluation of New Derivatives of Pyrido[1,2-a]benzimidazolic Ring System: The Pyrido[1',2':1,2]imidazo[4,5- $h]$ quinazolines
}

\author{
Marianne Dupuy,${ }^{a, b}$ Frederic Pinguet,${ }^{b}$ Olivier Chavignon, ${ }^{c}$ Jean-Michel Chezal, ${ }^{c}$ \\ Jean-Claude Teulade, ${ }^{c}$ Jean-Pierre ChaPat, ${ }^{a}$ and Yves Blache ${ }^{*}, a$
}

Laboratoire de Chimie organique Pharmaceutique, ${ }^{a}$ E.A. 2414, 15 Avenue Charles Flahault, Faculté de Pharmacie, 34060 Montpellier, Laboratoire d'oncopharmacologie, Centre Régional de Lutte contre le Cancer, ${ }^{b}$ Val d'Aurelle, 34298 Montpellier Cedex 05, and Laboratoire de Chimie Organique Pharmaceutique, UFR de Pharmacie, ${ }^{c} 28$ Place Henry Dunant, B.P. 38, 63001 Clermont-Ferrand, France. $\quad$ Received January 5, 2001; accepted May 21, 2001

\begin{abstract}
Access to the original series of pyrido[1',2':1,2]imidazo[4,5-h]quinazoline was developed from a 1,3-dicarbonyl unit with some "N-C-N" bisnucleophilic reagents and the derivatives obtained were evaluated for in vitro cytotoxic activities against $\mathrm{HL60}$ and A2780 cells. All compounds exhibited cytotoxic activities on resistant cell lines $\left(\mathrm{MDR}^{+}\right.$; HL60R and A2780R) with no resistance phenomena.
\end{abstract}

Key words pyrido $\left[1^{\prime}, 2^{\prime}: 1,2\right]$ imidazo $[4,5-h]$ quinazoline; anticancer agent; multidrug resistance

As a part of studies related to the pharmacochemistry of heterocycles with a bridgehead nitrogen, ${ }^{1)}$ we initiated a program aimed at examining the synthesis and cytotoxicity of new tetracyclic derivatives of azacarbazoles. ${ }^{2)}$ Since the pyrido[1,2-a]benzimidazole ring system has been found to exhibit anticancer properties by Badaway and Kappe, ${ }^{3)}$ a number of studies have been directed toward this heterocycle. These investigations showed good activities of the compounds substituted on ring A (Chart 1, compound type 1).

As a first approach to modifications which should be integrated into ring $\mathrm{C}$, we previously reported the regioselective synthesis of some pyrrolo ${ }^{4)}$ and pyrazolo ${ }^{5)}$ derivatives and their antitumor activities in vitro against resistant cell lines $\left.\left(\mathrm{MDR}^{+}\right) .{ }^{6}\right)$ To determine the effect of the nature of the heterocycle, we were interested in the pyrido[ $\left[1^{\prime}, 2^{\prime}: 1,2\right] \mathrm{imi}-$ dazo[4,5-h]quinazoline series (compound type 2 ). This class of compounds is related to the general class of tetracyclic angular heterocycles which are of great interest in the field of new potential anticancer agents. For example, a number of angular analogs of ellipticine ${ }^{7}$ have been prepared in this way. From these studies the most promising product appeared to be intoplicine $3{ }^{8)}$ which is actually in a phase 2 clinical trial. ${ }^{9)}$ In this context, we became interested in the synthesis of the original series of pyrido $\left[1^{\prime}, 2^{\prime}: 1,2\right]$ imidazo[4,5-h]quinazoline possessing a bridgehead nitrogen atom with a view to designing some new potential anticancer agents. In this paper, we describe the access to this framework, and our results concerning the cytotoxicity of the synthesized compounds against two human cell lines exhibiting the MDR phenotype. ${ }^{10)}$

Chemistry Retrosynthetic analysis showed that this series of compounds $\mathbf{4}$ was accessible by dehydrogenation of dihydro precursors 5 which can themself be obtained by reaction of an appropriate bisnucleophilic reagent with tricyclic 1,3-dicarbonyl units 6 possessing the tetrahydropyrido[1,2-a] benzimidazolic framework ${ }^{11)}$ (Chart 2).

The starting hydroxymethylene derivatives 13,14 were obtained according to the literature. ${ }^{5)}$ Compound $\mathbf{1 5}$ was prepared using the same procedure in two steps from 2-amino-5chloropyridine (Chart 3).

Access to the Pyrido[1,2:1',2']imidazo[4,5-h]quinazo- line Framework Our strategy based on a direct route to the pyrimidinic ring requiring the condensation of a bisnucleophilic " $\mathrm{N}-\mathrm{C}-\mathrm{N}$ " equivalent reagent with the 1,3-dicarbonyl units 13-15 led us to investigate first the reactivity of $\mathbf{1 3}$ toward a variety of such reagents. Results are summarized in Table 1.

The unsubstituted pyrimidine derivative $\mathbf{1 6}$ could be easily prepared by reaction of $\mathbf{1 3}$ with formamidinium acetate. For the synthesis of the 2 -amino derivative 17 , guanidinium chlo-
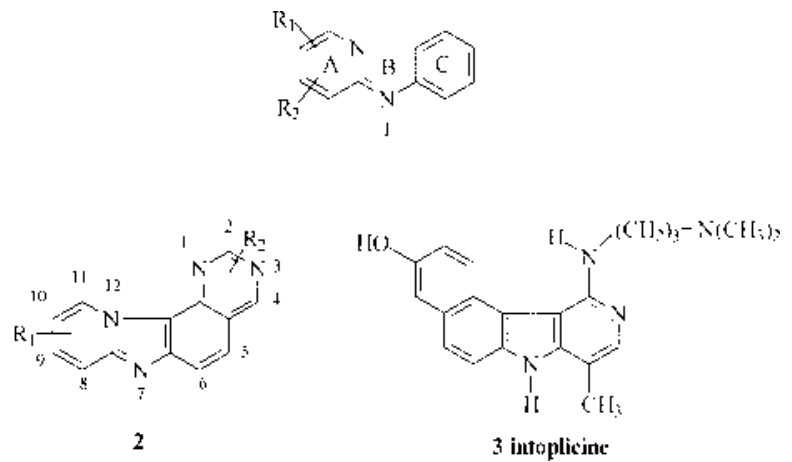

Chart 1

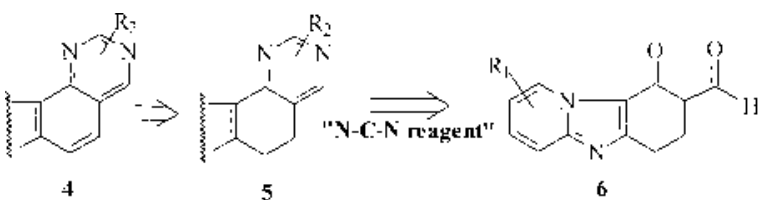

Chart 2

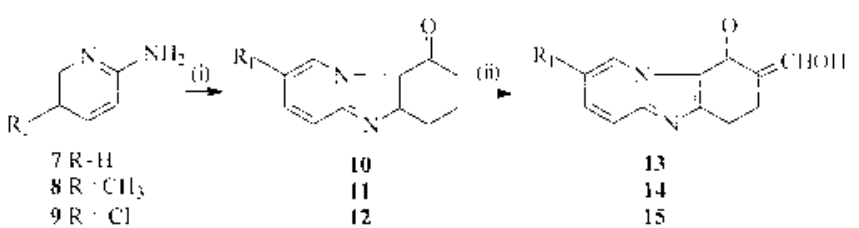

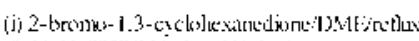

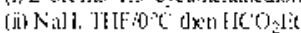

Chart 3 
Table 1. Access to the Pyrimidinic Framework from Compound $\mathbf{1 3}$

$\mathrm{N}-\mathrm{C}-\mathrm{N}$ reagent
and conditions

ride was used. Surprisingly, under the first condition used, the expected product was not obtained, but the 6,7,8,9tetrahydropyrido[1,2- $a$ ] benzimidazol-9-one $\mathbf{1 0}$ was isolated. Formation of $\mathbf{1 0}$ under these conditions was found to be the result of a retro-claisen type reaction performed with sodium ethylate generated from ethanol by sodium hydride. This hypothesis was confirmed by the following experiments. Treatment of $\mathbf{1 3}$ by $\mathrm{NaH}$ in ethanol led to the formation of $\mathbf{1 0}$, and the same result was obtained when treating 13 with sodium in ethanol while the treatment of $\mathbf{1 3}$ by $\mathrm{NaH}$ in THF gave no results. To prevent this reaction, compound $\mathbf{1 3}$ was allowed to react with guanidinium chloride in the presence of potassium carbonate in DMF, and the desired product 17 was obtained. Synthesis of the thiomethyl derivative $\mathbf{1 8}$ was finally investigated and first conducted by reaction of 13 with 2(methylthio)pseudourea in refluxing ethanol. Under these conditions, heterocyclization occurred to give $\mathbf{1 8}$ as the minor product admixed with the acetal 19. Formation of this acetal is probably catalyzed by sulfuric acid liberated in situ from 2-(methylthio)pseudourea sulfate. To avoid this reaction, the $\beta$-hydroxenone $\mathbf{1 3}$ was first converted to its benzoyl ester 20, which reacted with 2-(methylthio)pseudourea in ethanol to give the excepted product 18 with only $5 \%$ yield. ${ }^{12)}$ When the reaction was performed in DMF, under basic conditions $\left(\mathrm{Et}_{3} \mathrm{~N}\right)$, formation of $\mathbf{1 8}$ occurred in $55 \%$ yield (Chart 4 ).

Compounds 14 and 15 were also allowed to react with guanidinium acetate leading to the dihydropyrido[1', $\left.2^{\prime}: 1,2\right]$ imidazo[4,5-h]quinazolines 21, 22 with $61 \%$ and $46 \%$ yield respectively showing that yields are increased by the methyl group. Finally dehydrogenations of 16-18, 21, 22 were investigated by treating these derivatives with palladium on charcoal. The corresponding pyridoimidazoquinazolines 23-27 were obtained. The best yield was observed with the 2-amino derivative $\mathbf{2 4}$ while the 10-methyl derivative was obtained in only $7 \%$ yield (Chart 5 ).

All compounds were characterized by NMR experiments.

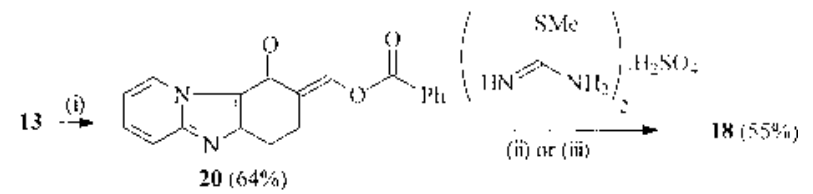

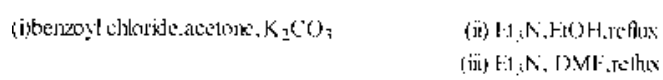

Chart 4



Chart 5

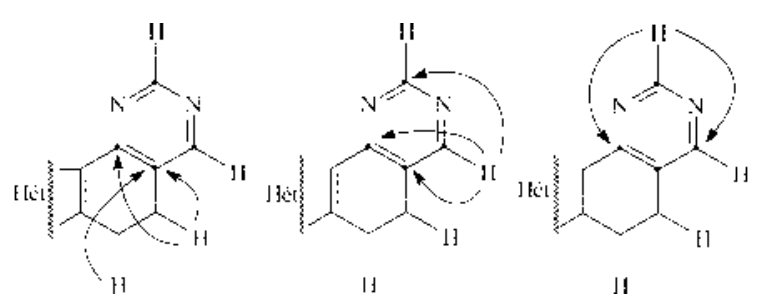

Fig. 1. ${ }^{1} \mathrm{H}^{-13} \mathrm{C}$ Long-Range Correlation Observed from INEPT Spectra of 16

In particular, in the cases of $\mathbf{1 6}$ and $\mathbf{2 3}$, assignment of the different signals of $\mathrm{H}-2$ and $\mathrm{H}-4$ (two singlets at 9.01 and $8.48 \mathrm{ppm}$ ) was made on the basis of selective INEPT experiments as illustrated in Fig. 1. Carbon C-12b was expected to show a significant long-range coupling with both protons $\mathrm{H}$ 2 and $\mathrm{H}-4$, while carbon $\mathrm{C}-4 \mathrm{a}$ would exhibit a long-range coupling only with H-4. As illustrated in Fig. 1, the unambigous attribution for $\mathrm{C}-12 \mathrm{~b}(154.49 \mathrm{ppm})$ and for $\mathrm{C}-4 \mathrm{a}$ (125.53) were obtained by selective irradiation of $\mathrm{H}-5$ causing polarization transfer to $\mathrm{C}-12 \mathrm{~b}$ and $\mathrm{C}-4 \mathrm{a}$, and of $\mathrm{H}-6$ (polarization transfer to $\mathrm{C}-4 \mathrm{a}$ ). Furthermore, selective irradiation of the signal at $8.48 \mathrm{ppm}$ showed the transfer of $\mathrm{C}-12 \mathrm{~b}$ and $\mathrm{C}-4 \mathrm{a}$, while the selective irradiation of the signal at $9.01 \mathrm{ppm}$ showed the transfer of $\mathrm{C}-12 \mathrm{~b}$ only; this signal was attributed to $\mathrm{H}-2$, while the signal at $8.48 \mathrm{ppm}$ was attributed to $\mathrm{H}-4$. Analogous correlations were observed for $\mathbf{2 3}$ leading to the following attributions: $\mathrm{H}-2$ at $9.38 \mathrm{ppm}$ and $\mathrm{H}-4$ at $9.42 \mathrm{ppm}$.

Pharmacology The cytotoxic properties of compounds 16, 21, 23 and 24 were evaluated by a cell growth inhibition assay against two human cell lines: HL60 (leukemia) and A2780 (ovarian). The resistant sublines HL60R and A2780R were established by the continuous spasm of cells to gradually increasing concentrations of daunorubicin and doxorubicin. All results are expressed as $\mathrm{IC}_{50}$ and reported in Tables 2 and 3.

\section{Results and Discussion}

All compounds exhibited cytotoxic activities against both HL60S and A2780S cell lines. In the case of the HL60 cell 
Table 2. Cytotoxicity of Compounds against the HL 60 Cell Line

\begin{tabular}{|c|c|c|c|c|c|}
\hline \multirow{2}{*}{ Compound } & \multicolumn{2}{|c|}{ HL60S } & \multicolumn{2}{|c|}{ HL60R } & \multirow{2}{*}{$\mathrm{RF}$} \\
\hline & $\mathrm{IC}_{50}$ & S.D. & $\mathrm{IC}_{50}$ & S.D. & \\
\hline Doxorubicin & $2.30 \times 10^{-6}$ & $0.51 \times 10^{-6}$ & $1.76 \times 10^{-4}$ & $0.93 \times 10^{-4}$ & 76 \\
\hline 16 & $1.60 \times 10^{-4}$ & $0.23 \times 10^{-4}$ & $1.60 \times 10^{-4}$ & $0.089 \times 10^{-4}$ & 1 \\
\hline 21 & $1.78 \times 10^{-3}$ & $0.64 \times 10^{-4}$ & $1.59 \times 10^{-3}$ & $0.46 \times 10^{-3}$ & 1 \\
\hline 23 & $1.90 \times 10^{-4}$ & $0.10 \times 10^{-4}$ & $1.70 \times 10^{-4}$ & $0.11 \times 10^{-4}$ & 1 \\
\hline 24 & $4.50 \times 10^{-4}$ & $0.90 \times 10^{-4}$ & $5.9 \times 10^{-4}$ & $0.08 \times 10^{-4}$ & 1.3 \\
\hline
\end{tabular}

S.D., standard deviation; RF, resistance factor.

Table 3. Cytotoxicity of Compounds against the A 2780 Cell Line

\begin{tabular}{|c|c|c|c|c|c|}
\hline \multirow{2}{*}{ Compound } & \multicolumn{2}{|c|}{ A2780S } & \multicolumn{2}{|c|}{ A2780R } & \multirow{2}{*}{$\mathrm{RF}$} \\
\hline & $\mathrm{IC}_{50}$ & S.D. & $\mathrm{IC}_{50}$ & S.D. & \\
\hline Doxorubicin & $0.34 \times 10^{-6}$ & $0.05 \times 10^{-6}$ & $0.28 \times 10^{-4}$ & $1.98 \times 10^{-6}$ & 82 \\
\hline 16 & $1.50 \times 10^{-4}$ & $0.18 \times 10^{-4}$ & $1.30 \times 10^{-4}$ & $0.32 \times 10^{-4}$ & 1 \\
\hline 21 & $1.75 \times 10^{-3}$ & $0.10 \times 10^{-3}$ & $1.34 \times 10^{-3}$ & $0.63 \times 10^{-4}$ & 1 \\
\hline 23 & $3.60 \times 10^{-5}$ & $0.12 \times 10^{-5}$ & $3.70 \times 10^{-5}$ & $0.15 \times 10^{-5}$ & 1 \\
\hline 24 & $5.10 \times 10^{-5}$ & $0.30 \times 10^{-5}$ & $5.80 \times 10^{-5}$ & $0.10 \times 10^{-5}$ & 1.1 \\
\hline
\end{tabular}

S.D., standard deviation; RF, resistance factor.

line, the less active compound was the 2-methyl derivative 21, while other compounds showed similar activities against both sensitive and resistant cells with small resistance factor (RF $c a$. 1 for 16, 23, 24 and 76 for doxorubicin). In addition, no effect of the dehydrogenation was observed on the activities.

The most interesting results were obtained on the A2780S and A2780R cell lines with the dehydrogenated compounds 23 and 24 which exhibited the highest activities. These activities were approximately 10 times better than their hydrogenated homologues and close to those observed for doxorubicine on the resistant cell line. In addition, in these two cases, the enhanced cytotoxicity did not induce the resistance phenomena (RF ca. 1).

Finally, these results showed that the pyrido $\left[1^{\prime}, 2^{\prime}: 1,2\right]$ imidazo[4,5-h]quinazoline skeleton is an interesting framework in which to study a new class of potential cytotoxic agents. As for the previously described pyrrolo and pyrazolo derivatives, ${ }^{4,5}$ the principal interest in these compounds resides in their activities on resistant cell lines with better activities of the six membered heterocyclic derivatives. Also the planerity of the molecules is necessary to enhance the cytotoxic properties.

\section{Conclusion}

In this paper, we have reported the synthesis of a new class of tetracyclic bridgehead nitrogen heterocycles: the pyrido $\left[1^{\prime}, 2^{\prime}: 1,2\right]$ imidazo[4,5- $\left.h\right]$ quinazoline framework. Reactivity of 1,3-dicarbonyl unit (13) derived from 6,7,8,9tetrahydropyrido[1,2-a]benzimidazol-9-one was reported toward a set of "N-C-N" bisnucleophilic reagents showing the influence of the reaction conditions on the reactivity of such systems. Further studies on the pharmacomodulation of such compounds are now in progress and should lead to an interesting class of new potential anticancer agents with no resistant phenomena.

\section{Experimental}

Melting points were determined on a Buchi capillary melting point apparatus and are not corrected. Elemental analysis was performed by Microanalytical Center, ENSCM, Montpellier. Spectral measurements were taken using the following instruments: ${ }^{1} \mathrm{H}-\mathrm{NMR}$ spectra were taken on a Brüker $\mathrm{AC} 100$ and a EM $400 \mathrm{WB}$ instrument; ${ }^{13} \mathrm{C}-\mathrm{NMR}$ spectra were obtained at $26^{\circ} \mathrm{C}$ with proton noise decoupling at $25 \mathrm{MHz}$ with a Brüker $\mathrm{AC} 100$ and a EM $400 \mathrm{WB}$ instrument. Chemical shifts are expressed relative to residual chloroform. Mass spectra were recorded on a LKB 2091 spectrometer at $15 \mathrm{eV}\left[\mathrm{q}(\right.$ source $\left.)=180^{\circ} \mathrm{C}\right]$.

2-Chloro-6,7,8,9-tetrahydropyrido[1,2-a]benzimidazol-9-one (12) This compound was obtained in $49 \%$ yield according to the methodology given in the literature (ref. 8) for the synthesis of $\mathbf{1 0}$ and 11. $\mathrm{mp}: 119$ $121^{\circ} \mathrm{C}$ (recrystallization solvent, methanol); MS m/z: 222 (38), 220 (100), 194 (27), 192 (73), 164 (32), 129 (38). ${ }^{1} \mathrm{H}-\mathrm{NMR}\left(\mathrm{CDCl}_{3}, 400 \mathrm{MHz}\right) \delta: 2.12$ $\left(\mathrm{t}, 2 \mathrm{H}, J_{6-7}=J_{7-8}=6.0 \mathrm{~Hz}, \mathrm{H}_{7}\right), 2.53\left(\mathrm{t}, 2 \mathrm{H}, \mathrm{H}_{8}\right), 2.93\left(\mathrm{t}, 2 \mathrm{H}, \mathrm{H}_{6}\right), 7.29(\mathrm{dd}$, $\left.1 \mathrm{H}, J_{3-4}=9.0 \mathrm{~Hz}, J_{1-3}=1.5 \mathrm{~Hz}, \mathrm{H}_{3}\right), 7.47\left(\mathrm{dd}, 1 \mathrm{H}, J_{1-4}=1.1 \mathrm{~Hz}, \mathrm{H}_{4}\right), 9.16$ $\left(\mathrm{s}, 1 \mathrm{H}, \mathrm{H}_{1}\right) \cdot{ }^{13} \mathrm{C}-\mathrm{NMR}\left(\mathrm{CDCl}_{3}, 100 \mathrm{MHz}\right) \delta: 23.5\left(\mathrm{C}_{6}\right), 25.2\left(\mathrm{C}_{7}\right), 38.1\left(\mathrm{C}_{8}\right)$, $116.8\left(\mathrm{C}_{4}\right), 119.5\left(\mathrm{C}_{9 \mathrm{a}}\right), 122.4\left(\mathrm{C}_{2}\right), 126.0\left(\mathrm{C}_{1}\right), 130.2\left(\mathrm{C}_{3}\right), 145.9\left(\mathrm{C}_{5 \mathrm{a}}\right)$, $160.3\left(\mathrm{C}_{4 \mathrm{a}}\right), 188.2(\mathrm{C}=\mathrm{O})$. Anal. Calcd for $\mathrm{C}_{11} \mathrm{H}_{9} \mathrm{~N}_{2} \mathrm{OCl}$ : C, 59.88; H, 4.11; N, 12.70. Found: C, 59.75; H, 4.21; N, 12.51 .

2-Chloro-8-hydroxymethylene-6,7,8,9-tetrahydropyrido[1,2-a]benzimidazol-9-one (15) This compound was obtained in 74\% yield according to the methodology given in the literature (ref. 5) for the synthesis of $\mathbf{1 0}$ and 11. $\mathrm{mp}: 226-228^{\circ} \mathrm{C}$ (recrystallization solvent, methanol). MS m/z: 250 (26), 248 (71), 221 (30), 220 (48), 219 (100), 191 (17), 155 (26), 129 (13). ${ }^{1} \mathrm{H}-\mathrm{NMR}\left(\mathrm{CDCl}_{3}, 100 \mathrm{MHz}\right) \delta: 2.68\left(\mathrm{t}, 2 \mathrm{H}, J_{6-7}=6.0 \mathrm{~Hz}, \mathrm{H}_{7}\right), 3.07(\mathrm{t}, 2 \mathrm{H}$, $\left.\mathrm{H}_{6}\right), 7.27(\mathrm{~s}, 1 \mathrm{H}, \underline{\mathrm{CHOH}}), 7.45\left(\mathrm{dd}, 1 \mathrm{H}, J_{3-4}=9.4 \mathrm{~Hz}, J_{1-3}=1.9 \mathrm{~Hz}, \mathrm{H}_{3}\right)$, $7.69\left(\mathrm{~d}, 1 \mathrm{H}, \mathrm{H}_{4}\right), 9.35\left(\mathrm{~s}, 1 \mathrm{H}, \mathrm{H}_{1}\right) .{ }^{13} \mathrm{C}-\mathrm{NMR}\left(\mathrm{CDCl}_{3}, 25 \mathrm{MHz}\right) \delta_{\mathrm{CH}}: 23.8$ $\left(\mathrm{C}_{6}\right), 24.5\left(\mathrm{C}_{7}\right), 98.8(\mathrm{CHOH}), 116.7\left(\mathrm{C}_{4}\right), 126.2\left(\mathrm{C}_{1}\right), 131.4\left(\mathrm{C}_{3}\right), 159.7\left(\mathrm{C}_{2}\right)$. Anal. Calcd for $\mathrm{C}_{12} \mathrm{H}_{9} \mathrm{~N}_{2} \mathrm{O}_{2} \mathrm{Cl}$ : C, 57.96; H, 3.65; N, 11.27. Found: C, 57.79; H, 3.81; N, 11.11.

5,6-Dihydropyrido $\left[1^{\prime}, 2^{\prime}: 1,2\right]$ imidazo $[4,5-h] q u i n a z o l i n e ~(16)$ To a solution of $13(600 \mathrm{mg}, 2.8 \mathrm{mmol})$ in dry ethanol $(53 \mathrm{ml})$ was added $3.5 \mathrm{~g}$ (33.6 mmol) of formamidinium acetate. The solution was refluxed for $25 \mathrm{~h}$ under a nitrogen stream. After evaporation of solvent, the crude product was chromatographed on silica gel eluting with $\mathrm{CH}_{2} \mathrm{Cl}_{2} / \mathrm{CH}_{3} \mathrm{OH}(97 / 3)$ to give 16 (48\%). mp: $103-105^{\circ} \mathrm{C}$ (recrystallization solvent, methanol). MS m/z: 222 (100), 221 (70), 194 (20), 167 (20). ${ }^{1} \mathrm{H}-\mathrm{NMR}\left(\mathrm{CDCl}_{3}, 400 \mathrm{MHz}\right) \delta: 3.21(\mathrm{~m}$, $\left.4 \mathrm{H}, \mathrm{H}_{5}, \mathrm{H}_{6}\right), 7.07\left(\mathrm{t}, 1 \mathrm{H}, J_{10-11}=J_{10-9}=7.0 \mathrm{~Hz}, \mathrm{H}_{10}\right), 7.43\left(\mathrm{t}, 1 \mathrm{H}, \mathrm{H}_{9}\right), 7.72$ $\left(\mathrm{d}, 1 \mathrm{H}, J_{8-9}=7.0 \mathrm{~Hz}, \mathrm{H}_{8}\right), 8.48\left(\mathrm{~s}, 1 \mathrm{H}, \mathrm{H}_{4}\right), 9.01\left(\mathrm{~s}, 1 \mathrm{H}, \mathrm{H}_{2}\right), 9.58(\mathrm{~d}, 1 \mathrm{H}$, $\left.\mathrm{H}_{11}\right) .{ }^{13} \mathrm{C}-\mathrm{NMR}\left(\mathrm{CDCl}_{3}, 100 \mathrm{MHz}\right) \delta: 23.3\left(\mathrm{C}_{6}\right), 25.2\left(\mathrm{C}_{5}\right), 113.7\left(\mathrm{C}_{10}\right)$, $117.1\left(\mathrm{C}_{8}\right), 117.2(\mathrm{C}-12 \mathrm{a}), 125.5(\mathrm{C}-4 \mathrm{a}), 127.2\left(\mathrm{C}_{9}\right), 128.2(\mathrm{C}-11), 148.2(\mathrm{C}-$ 7a), 152.6 (C-6a), $153.3\left(\mathrm{C}_{4}\right), 154.5(\mathrm{C}-12 \mathrm{~b}), 156.9\left(\mathrm{C}_{2}\right)$. Anal. Calcd for 
$\mathrm{C}_{13} \mathrm{H}_{10} \mathrm{~N}_{4} \mathrm{OCl}: \mathrm{C}, 70.26 ; \mathrm{H}, 4.54 ; \mathrm{N}, 25.21$. Found: C, 70.41; H, 4.44; N, 25.15 .

2-Amino-5,6-dihydropyrido $\left[1^{\prime}, 2^{\prime}: 1,2\right]$ imidazo[4,5-h]quinazoline (17) To a stirred solution of $1.62 \mathrm{~g}(16.8 \mathrm{mmol})$ of guanidinium chloride and potassium carbonate in dry DMF $(20 \mathrm{ml})$ was added $200 \mathrm{mg}(0.9 \mathrm{mmol})$ of 13. The solution was refluxed for $2 \mathrm{~h}$. After filtration and evaporation of solvent the crude product was chromatographed on silica gel eluting with $\mathrm{CH}_{2} \mathrm{Cl}_{2} / \mathrm{CH}_{3} \mathrm{OH}\left(97 / 3\right.$ ). Yield: $53 \%$. mp: $239-241^{\circ} \mathrm{C}$ (recrystallization solvent, ethanol). MS $m / z$ : 238 (25), 237 (100), 236 (86), 209 (18). ${ }^{1} \mathrm{H}-\mathrm{NMR}$ $\left(\mathrm{CDCl}_{3}, 100 \mathrm{MHz}\right) \delta: 3.08\left(\mathrm{~m}, 4 \mathrm{H}, \mathrm{H}_{5}, \mathrm{H}_{6}\right), 5.35\left(\mathrm{NH}_{2}\right), 6.99(\mathrm{t}, 1 \mathrm{H}$, $\left.J_{9-10}=J_{10-11}=7.0 \mathrm{~Hz}, \mathrm{H}_{10}\right), 7.38\left(\mathrm{dt}, 1 \mathrm{H}, J_{9-8}=7.0 \mathrm{~Hz}, J_{9-11}=1.0 \mathrm{~Hz}, \mathrm{H}_{9}\right)$, $7.64\left(\mathrm{dd}, 1 \mathrm{H}, J_{8-10}=1.0 \mathrm{~Hz}, \mathrm{H}_{8}\right), 7.98\left(\mathrm{~s}, 1 \mathrm{H}, \mathrm{H}_{4}\right), 9.46\left(\mathrm{dd}, 1 \mathrm{H}, \mathrm{H}_{11}\right) \cdot{ }^{13} \mathrm{C}-$ NMR $\left(\mathrm{CDCl}_{3}, 25 \mathrm{MHz}\right) \delta: 23.5\left(\mathrm{C}_{6}\right), 24.2\left(\mathrm{C}_{5}\right), 113.6\left(\mathrm{C}_{10}\right), 115.5(\mathrm{C}-$ 12a), $116.7\left(\mathrm{C}_{8}\right), 122.6(\mathrm{C}-4 \mathrm{a}), 127.3\left(\mathrm{C}_{9}\right), 128.2\left(\mathrm{C}_{11}\right), 147.6(\mathrm{C}-7 \mathrm{a}), 152.7$ (C-6a), $153.2\left(\mathrm{C}_{4}\right), 155.9(\mathrm{C}-12 \mathrm{~b}), 161.3(\mathrm{C}-2)$. Anal. Calcd for $\mathrm{C}_{13} \mathrm{H}_{11} \mathrm{~N}_{5}: \mathrm{C}$, $65.81 ; \mathrm{H}, 4.67 ; \mathrm{N}, 29.52$. Found: C, 65.89; H, 4.71; N, 29.40.

2-(Methylthio)-5,6-dihydropyrido[$\left[1^{\prime}, 2^{\prime}: 1,2\right]$ imidazo[4,5- $\left.h\right]$ quinazoline (18) Method A: To a solution of $\mathbf{1 3}(500 \mathrm{mg}, 2.3 \mathrm{mmol})$ and $0.20 \mathrm{ml}$ of triethylamine in anhydrous EtOH $(30 \mathrm{ml})$ was added $5.2 \mathrm{~g}$ of methylthiopseudourea sulfate $(18.72 \mathrm{mmol})$. The solution was refluxed for $10 \mathrm{~h}$ under a nitrogen stream. After evaporation of solvent, the crude mixture was chromatographed on silica gel eluted with $\mathrm{CH}_{2} \mathrm{Cl}_{2} / \mathrm{CH}_{3} \mathrm{OH}(98 / 2)$ to give $\mathbf{1 8}$ in $5 \%$ yield. mp: $92-94^{\circ} \mathrm{C}$ (recrystallization solvent, ethylacetate). MS $\mathrm{m} / \mathrm{z}$ : 268 (100), 267 (20), 222 (40), 221 (56), 194 (28). ${ }^{1} \mathrm{H}-\mathrm{NMR}\left(\mathrm{CDCl}_{3}, 100\right.$ MHz) $\delta: 2.55\left(\mathrm{~s}, 3 \mathrm{H}, \mathrm{CH}_{3}\right), 3.02\left(\mathrm{~m}, 4 \mathrm{H}, \mathrm{H}_{5}, \mathrm{H}_{6}\right), 6.91\left(\mathrm{t}, 1 \mathrm{H}, J_{9-10}=\right.$ $\left.J_{10-11}=6.0 \mathrm{~Hz}, \mathrm{H}_{10}\right), 7.29\left(\mathrm{dt}, 1 \mathrm{H}, J_{8-9}=6.0 \mathrm{~Hz}, J_{9-11}=1.3 \mathrm{~Hz}, \mathrm{H}_{9}\right), 7.55(\mathrm{~d}$, $\left.1 \mathrm{H}, \mathrm{H}_{8}\right), 8.11\left(\mathrm{~s}, 1 \mathrm{H}, \mathrm{H}_{4}\right), 9.23\left(\mathrm{~d}, 1 \mathrm{H}, \mathrm{H}_{11}\right) .{ }^{13} \mathrm{C}-\mathrm{NMR}\left(\mathrm{CDCl}_{3}, 25 \mathrm{MHz}\right)$ $\delta_{\mathrm{CH}}: 13.9\left(\mathrm{CH}_{3}\right), 22.7\left(\mathrm{C}_{6}\right), 24.2\left(\mathrm{C}_{5}\right), 114.0\left(\mathrm{C}_{10}\right), 116.4\left(\mathrm{C}_{8}\right), 127.8(2 \mathrm{C}$, $\left.\mathrm{C}_{9}, \mathrm{C}_{11}\right), 153.2\left(\mathrm{C}_{4}\right)$. Anal. Calcd for $\mathrm{C}_{14} \mathrm{H}_{12} \mathrm{~N}_{4} \mathrm{~S}: \mathrm{C}, 62.67 ; \mathrm{H}, 4.51 ; \mathrm{N}$, 20.88. Found: $\mathrm{C}, 62.85 ; \mathrm{H}, 4.31 ; \mathrm{N}, 20.76$. Further elution yielded 8-diethoxymethyl-6,7,8,9-tetrahydropyrido[1,2-a]benzimidazol-9-one (19) with 48\% yield (brown oil). MS m/z: 288 (5), 259 (44), 213 (11), 185 (25), 157 (30), 103 (100). ${ }^{1} \mathrm{H}-\mathrm{NMR}\left(\mathrm{CDCl}_{3}, 100 \mathrm{MHz}\right) \delta: 1.22\left(\mathrm{~m}, 6 \mathrm{H}, \underline{\mathrm{CH}}_{3}-\mathrm{CH}_{2}\right)$, $2.35\left(\mathrm{t}, 2 \mathrm{H}, J_{6-7}=J_{7-8}=6.1 \mathrm{~Hz}, \mathrm{H}_{7}\right), 2.60-3.30\left(\mathrm{~m}, 3 \mathrm{H}, \mathrm{H}_{6}, \mathrm{H}_{8}\right), 3.61(\mathrm{~m}$, $\left.4 \mathrm{H}, \mathrm{CH}_{3}-\mathrm{CH}_{2}\right), 5.1\left(\mathrm{~s}, 1 \mathrm{H}, \mathrm{H}_{1^{\prime}}\right), 6.95\left(\mathrm{t}, 1 \mathrm{H}, J_{1-2}=J_{2-3}=7.0 \mathrm{~Hz}, \mathrm{H}_{2}\right), 7.47$ $\left(\mathrm{t}, 1 \mathrm{H}, J_{3-4}=7.0 \mathrm{~Hz}, \mathrm{H}_{3}\right), 7.59\left(\mathrm{~d}, 1 \mathrm{H}, \mathrm{H}_{4}\right), 9.21\left(\mathrm{~d}, 1 \mathrm{H}, \mathrm{H}_{1}\right),{ }^{13} \mathrm{C}-\mathrm{NMR}$ $\left(\mathrm{CDCl}_{3}, 25 \mathrm{MHz}\right) \delta: 15.2\left(\underline{\mathrm{CH}}_{3}-\mathrm{CH}_{2}\right) ; 15.32\left(\mathrm{CH}_{3}-\mathrm{CH}_{2}\right), 22.5\left(\mathrm{C}_{6}\right), 24.3$ $\left(\mathrm{C}_{7}\right), 51.1\left(\mathrm{C}_{8}\right), 63.6\left(\mathrm{CH}_{3}-\mathrm{CH}_{2}\right), 64.9\left(\mathrm{CH}_{3}-\mathrm{CH}_{2}\right), 102.2\left(\mathrm{C}_{1}\right), 114.3\left(\mathrm{C}_{2}\right)$, $116.8\left(\mathrm{C}_{4}\right), 119.8\left(\mathrm{C}_{9 \mathrm{a}}\right), 128.2\left(\overline{\mathrm{C}}_{1}\right), 129.4\left(\mathrm{C}_{3}\right), 148.1\left(\mathrm{C}_{5 \mathrm{a}}\right), 160.6\left(\mathrm{C}_{4 \mathrm{a}}\right), 187$. $1(\mathrm{C}=\mathrm{O})$. Anal. Calcd for $\mathrm{C}_{15} \mathrm{H}_{20} \mathrm{~N}_{2} \mathrm{O}_{3}: \mathrm{C}, 65.20 ; \mathrm{H}, 7.30 ; \mathrm{N}, 10.14$. Found: $\mathrm{C}, 65.31 ; \mathrm{H}, 7.23 ; \mathrm{N}, 10.25$.

Method B: To a solution of $20(500 \mathrm{mg}, 1.56 \mathrm{mmol})$ and $0.26 \mathrm{ml}$ of triethylamine in anhydrous DMF $(30 \mathrm{ml})$ was added $5.2 \mathrm{~g}$ of methylthiopseudourea sulfate $(18.72 \mathrm{mmol})$. The solution was refluxed for $23 \mathrm{~h}$ under a nitrogen stream. After evaporation of solvent, the crude mixture was chromatographed on silica gel eluted with $\mathrm{CH}_{2} \mathrm{Cl}_{2} / \mathrm{CH}_{3} \mathrm{OH}$ (98/2) to give $\mathbf{1 8}$ in $55 \%$ yield.

8-[(Benzoyloxy)methylene]-6,7,8,9-tetrahydropyrido[1,2- $a$ ]benzimidazol-9-one (20) To a solution of $\mathbf{1 3}(1 \mathrm{~g}, 4.7 \mathrm{mmol})$ and potassium carbonate $(0.78 \mathrm{~g}, 5.6 \mathrm{mmol})$ in dry acetone $(67 \mathrm{ml})$ was added $1.62 \mathrm{ml}(14.1 \mathrm{mmol})$ of benzoyl chloride over $20 \mathrm{~min}$. The solution was stirred at room temperature under a nitrogen stream overnight. After evaporation of solvent, the crude product was taken up in $\mathrm{CH}_{2} \mathrm{Cl}_{2}$ and poured over ice. The layers were separated and the organic layer was washed with $10 \% \mathrm{NaHCO}_{3}$ and brine, dried over $\mathrm{Na}_{2} \mathrm{SO}_{4}$ and concentrated. Yield: $64 \%$; mp: $189-191^{\circ} \mathrm{C}$ (recrystallization solvent, ether). MS m/z: 318 (34), 213 (13), 185 (14), 105 (100). ${ }^{1} \mathrm{H}-\mathrm{NMR}\left(\mathrm{CDCl}_{3}, 100 \mathrm{MHz}\right) \delta: 3.16\left(\mathrm{~m}, 4 \mathrm{H}, \mathrm{H}_{6}, \mathrm{H}_{7}\right), 7.02\left(\mathrm{t}, 1 \mathrm{H}, J_{1-2}=\right.$ $\left.J_{2-3}=7.0 \mathrm{~Hz}, \mathrm{H}_{2}\right), 7.53\left(\mathrm{~m}, 5 \mathrm{H}, \mathrm{H}_{\mathrm{Ar}}\right), 8.10\left(\mathrm{~m}, 2 \mathrm{H}, \mathrm{H}_{3}, \mathrm{H}_{4}\right), 8.52\left(\mathrm{~s}, 1 \mathrm{H}, \mathrm{H}_{1^{\prime}}\right)$, $9.29\left(\mathrm{~d}, 1 \mathrm{H}, \mathrm{H}_{1}\right) \cdot{ }^{13} \mathrm{C}-\mathrm{NMR}\left(\mathrm{CDCl}_{3}, 25 \mathrm{MHz}\right) \delta: 22.7\left(\mathrm{C}_{7}\right), 23.9\left(\mathrm{C}_{6}\right), 114.2$ $\left(\mathrm{C}_{2}\right), 116.4\left(\mathrm{C}_{4}\right), 119.8\left(\mathrm{C}_{9 \mathrm{a}}\right), 120.7\left(\mathrm{C}_{8}\right), 127.9\left(\mathrm{C}_{\mathrm{Ar}}\right), 128.0\left(\mathrm{C}_{\mathrm{Ar}}\right), 128.3(\mathrm{C} 2$, $\left.\mathrm{C}_{\mathrm{Ar}}\right), 129.4\left(\mathrm{C}_{1}{ }^{*}\right), 129.8\left(2 \mathrm{C}, \mathrm{C}_{\mathrm{Ar}}\right), 133.7\left(\mathrm{C}_{3}{ }^{*}\right), 140.3\left(\mathrm{C}_{1}\right), 147.9\left(\mathrm{C}_{5 \mathrm{a}}\right)$, $158.9\left(\mathrm{C}_{4 a}\right), 176.8(\mathrm{C}=\mathrm{O})$. Anal. Calcd for $\mathrm{C}_{19} \mathrm{H}_{14} \mathrm{~N}_{2} \mathrm{O}_{3}: \mathrm{C}, 71.69 ; \mathrm{H}, 4.43$; $\mathrm{N}, 8.80$. Found: $\mathrm{C}, 71.75 ; \mathrm{H}, 4.26 ; \mathrm{N}, 8.99$.

10-Methyl-5,6-dihydropyrido $\left[1^{\prime}, 2^{\prime}: 1,2\right]$ imidazo[4,5-h]quinazoline (21) This compound was obtained according to the procedure used for $\mathbf{1 6}$ (reaction time, $24 \mathrm{~h}$ ); chromatography: silica gel, $\mathrm{CH}_{2} \mathrm{Cl}_{2} / \mathrm{CH}_{3} \mathrm{OH}$ (95/5); yield: $61.5 \%$. mp: $207-209^{\circ} \mathrm{C}$ (recrystallization solvent, methanol). MS m/z: 236 (100), 235 (66), 208 (7), 181 (7). ${ }^{1} \mathrm{H}-\mathrm{NMR}\left(\mathrm{CDCl}_{3}, 100 \mathrm{MHz}\right) \delta: 2.39$ (s, $\left.\mathrm{CH}_{3}\right), 3.08\left(\mathrm{~m}, 4 \mathrm{H}, \mathrm{H}_{5}, \mathrm{H}_{6}\right), 7.17\left(\mathrm{~d}, 1 \mathrm{H}, J_{8-9}=9.0 \mathrm{~Hz}, \mathrm{H}_{9}\right), 7.51\left(\mathrm{~d}, 1 \mathrm{H}, \mathrm{H}_{8}\right)$, $8.36\left(\mathrm{~s}, 1 \mathrm{H}, \mathrm{H}_{4}\right), 8.89\left(\mathrm{~s}, 1 \mathrm{H}, \mathrm{H}_{2}\right), 9.20\left(\mathrm{~s}, 1 \mathrm{H}, \mathrm{H}_{11}\right) \cdot{ }^{13} \mathrm{C}-\mathrm{NMR}\left(\mathrm{CDCl}_{3}\right.$, $25 \mathrm{MHz}) \delta: 18.07\left(\mathrm{CH}_{3}\right), 22.7\left(\mathrm{C}_{6}\right), 24.7\left(\mathrm{C}_{5}\right), 115.7\left(\mathrm{C}_{8}\right), 116.3(\mathrm{C}-12 \mathrm{a})$, $123.4(\mathrm{C}-10), 125.1(\mathrm{C}-4 \mathrm{a}), 125.7\left(\mathrm{C}_{9}\right), 129.9\left(\mathrm{C}_{11}\right), 148.6(\mathrm{C}-7 \mathrm{a}), 151.6(\mathrm{C}-$ 6a), $152.5\left(\mathrm{C}_{4}\right), 154.1(\mathrm{C}-12 \mathrm{~b}), 156.3\left(\mathrm{C}_{2}\right)$. Anal. Calcd for $\mathrm{C}_{14} \mathrm{H}_{12} \mathrm{~N}_{4}$ : C, 71.17; H, 5.12; N, 23.71. Found: C, 71.25; H, 5.22; N, 23.53.
10-Chloro-5,6-dihydropyrido $\left[\mathbf{1}^{\prime}, \mathbf{2}^{\prime}: \mathbf{1 , 2}\right]$ imidazo $[4,5-h]$ quinazoline (22) This compound was obtained in $46 \%$ yield according to the procedure used for 16 (reaction time, 24h); chromatography: silica gel eluted with $\mathrm{CH}_{2} \mathrm{Cl}_{2} / \mathrm{CH}_{3} \mathrm{OH}(95 / 5)$. mp: $185-187^{\circ} \mathrm{C}$. MS m/z 256 (97), 255 (42), 220 (22), 149 (15). ${ }^{1} \mathrm{H}-\mathrm{NMR}\left(\mathrm{CDCl}_{3}, 100 \mathrm{MHz}\right) \delta: 3.20\left(\mathrm{~m}, 4 \mathrm{H}, \mathrm{H}_{5}, \mathrm{H}_{6}\right), 7.33(\mathrm{~d}$, $\left.1 \mathrm{H}, \mathrm{H}_{9}, J_{8-9}=1.2 \mathrm{~Hz}\right), 7.62\left(\mathrm{~d}, 1 \mathrm{H}, \mathrm{H}_{8}\right), 8.49\left(\mathrm{~s}, 1 \mathrm{H}, \mathrm{H}_{4}\right), 9.00\left(\mathrm{~s}, 1 \mathrm{H}, \mathrm{H}_{2}\right)$, $9.61\left(\mathrm{~s}, 1 \mathrm{H}, \mathrm{H}_{11}\right)$. Anal. Calcd for $\mathrm{C}_{13} \mathrm{H}_{9} \mathrm{~N}_{4} \mathrm{Cl}: \mathrm{C}, 60.83 ; \mathrm{H}, 3.53 ; \mathrm{N}, 21.83$. Found: C, 60.75; H, 3.36; N, 21.99 .

Pyrido[1', $\left.\mathbf{1}^{\prime}: \mathbf{1}, 2\right]$ imidazo[4,5-h]quinazoline (23) To a solution of $\mathbf{1 6}$ $(300 \mathrm{mg}, 1.35 \mathrm{mmol})$ in diphenylether $(6.0 \mathrm{ml})$ was added $140 \mathrm{mg}$ of palladium $(10 \%)$ on charcoal. The solution was refluxed for $22 \mathrm{~h}$. After filtration of the palladium, the crude product was washed with methanol and chromatographed on silica gel with $\mathrm{CH}_{2} \mathrm{Cl}_{2} / \mathrm{CH}_{3} \mathrm{OH}(99 / 1)$. Yield: $34 \%$. mp: $186-188^{\circ} \mathrm{C}$ (recrystallization solvent, ethanol). MS m/z: 220 (100), 193 (12), 166 (14). ${ }^{1} \mathrm{H}-\mathrm{NMR}\left(\mathrm{CDCl}_{3}, 400 \mathrm{MHz}\right) \delta$ : $7.15\left(\mathrm{t}, 1 \mathrm{H}, J_{9-10}=J_{10-11}=\right.$ $\left.7.0 \mathrm{~Hz}, \mathrm{H}_{10}\right), 7.63\left(\mathrm{t}, 1 \mathrm{H}, J_{8-9}=7.0 \mathrm{~Hz}, \mathrm{H}_{9}\right), 7.85\left(\mathrm{~d}, 1 \mathrm{H}, J_{5-6}=8.8 \mathrm{~Hz}, \mathrm{H}_{5}\right)$, $7.90\left(\mathrm{~d}, 1 \mathrm{H}, \mathrm{H}_{8}\right), 8.06\left(\mathrm{~d}, 1 \mathrm{H}, \mathrm{H}_{6}\right), 9.38\left(\mathrm{~s}, 1 \mathrm{H}, \mathrm{H}_{2}\right), 9.42\left(\mathrm{~s}, 1 \mathrm{H}, \mathrm{H}_{4}\right), 9.98(\mathrm{~d}$, $\left.1 \mathrm{H}, \mathrm{H}_{11}\right) \cdot{ }^{13} \mathrm{C}-\mathrm{NMR}\left(\mathrm{CDCl}_{3}, 100 \mathrm{MHz}\right) \delta: 112.8\left(\mathrm{C}_{10}\right), 117.6\left(\mathrm{C}_{8}\right), 120.2$ $\left(\mathrm{C}_{12 \mathrm{a}}\right), 121.2\left(\mathrm{C}_{4 \mathrm{a}}\right), 122.4\left(\mathrm{C}_{6}\right), 124.8\left(\mathrm{C}_{5}\right), 130.0\left(\mathrm{C}_{9}\right), 130.1\left(\mathrm{C}_{11}\right), 142.5$ $\left(\mathrm{C}_{12 \mathrm{~b}}\right), 148.3\left(\mathrm{C}_{6 \mathrm{a}}\right), 149.5\left(\mathrm{C}_{7 \mathrm{a}}\right), 155.4\left(\mathrm{C}_{2}\right), 158.6\left(\mathrm{C}_{4}\right)$. Anal. Calcd for $\mathrm{C}_{13} \mathrm{H}_{8} \mathrm{~N}_{4}: \mathrm{C}, 70.90 ; \mathrm{H}, 3.66 ; \mathrm{N}, 25.44$. Found: $\mathrm{C}, 71.02 ; \mathrm{H}, 3.58 ; \mathrm{N}, 25.40$.

2-Aminopyrido[ $\left[\mathbf{1}^{\prime}, \mathbf{2}^{\prime}: \mathbf{1}, \mathbf{2}\right]$ imidazo[4,5-h]quinazoline (24) This compound was obtained according to the procedure used for $\mathbf{2 3}$ (reaction time, $6 \mathrm{~h}$ ); chromatography: silica gel with $\mathrm{CH}_{2} \mathrm{Cl}_{2} / \mathrm{CH}_{3} \mathrm{OH}$ (98/2). Yield: $67 \%$. $\mathrm{mp}>260^{\circ} \mathrm{C}$ (recrystallization solvent, methanol). MS m/z: 236 (100), 235 (70), 209 (23), 208 (23). ${ }^{1} \mathrm{H}-\mathrm{NMR}\left(\mathrm{CDCl}_{3}, 100 \mathrm{MHz}\right) \delta: 8.00(\mathrm{t}, 1 \mathrm{H}$, $\left.J_{10-11}=J_{9-10}=7.0 \mathrm{~Hz}, \mathrm{H}_{10}\right), 8.41\left(\mathrm{t}, 1 \mathrm{H}, \mathrm{H}_{9}\right), 8.50\left(\mathrm{~m}, 3 \mathrm{H}, \mathrm{H}_{8}, \mathrm{H}_{5}, \mathrm{H}_{6}\right), 9.85$ $\left(\mathrm{s}, 1 \mathrm{H}, \mathrm{H}_{4}\right), 10.81\left(\mathrm{~d}, 1 \mathrm{H}, \mathrm{H}_{11}\right) \cdot{ }^{13} \mathrm{C}-\mathrm{NMR}\left(\mathrm{CDCl}_{3}, 25 \mathrm{MHz}\right) \delta_{\mathrm{CH}}: 112.3$ $\left(\mathrm{C}_{10}\right), 115.2\left(\mathrm{C}_{8}\right), 115.9\left(\mathrm{C}_{6}\right), 125.7\left(\mathrm{C}_{5}\right), 129.7\left(\mathrm{C}_{9}{ }^{*}\right), 130.2\left(\mathrm{C}_{11}{ }^{*}\right), 160.2$ $\left(\mathrm{C}_{4}\right)$. Anal. Calcd for $\mathrm{C}_{13} \mathrm{H}_{9} \mathrm{~N}_{5}: \mathrm{C}, 66.37 ; \mathrm{H}, 3.86 ; \mathrm{N}, 29.77$. Found: $\mathrm{C}, 66.45$; $\mathrm{H}, 3.87 ; \mathrm{N}, 29.68$.

2-(Methylthio)pyrido $\left[1^{\prime}, 2^{\prime}: 1,2\right]$ imidazo[4,5-h]quinazoline (25) This compound was obtained according to the procedure used for $\mathbf{2 3}$ (reaction time, $10 \mathrm{~h}$ ); chromatography: silica gel with $\mathrm{CH}_{2} \mathrm{Cl}_{2}$. Yield: $24 \%$; mp: $196-$ $198{ }^{\circ} \mathrm{C}$ (recrystallization solvent, ethyl acetate). MS $m / z: 266$ (100), 221 (15), 220 (73), 219 (37), 193 (26), 166 (15). ${ }^{1} \mathrm{H}-\mathrm{NMR}\left(\mathrm{CDCl}_{3}, 100 \mathrm{MHz}\right) \delta$ : $2.58\left(\mathrm{~s}, \mathrm{CH}_{3}\right), 6.88\left(\mathrm{t}, 1 \mathrm{H}, J_{9-10}=J_{10-11}=6.6 \mathrm{~Hz}, \mathrm{H}_{10}\right), 7.30-7.75(\mathrm{~m}, 4 \mathrm{H}$, $\left.\mathrm{H}_{5}, \mathrm{H}_{6}, \mathrm{H}_{8}, \mathrm{H}_{9}\right), 8.84\left(\mathrm{~s}, 1 \mathrm{H}, \mathrm{H}_{4}\right), 9.29\left(\mathrm{~d}, 1 \mathrm{H}, \mathrm{H}_{11}\right) .{ }^{13} \mathrm{C}-\mathrm{NMR}\left(\mathrm{CDCl}_{3}\right.$, $25 \mathrm{MHz}) \delta_{\mathrm{CH}}: 14.4\left(\mathrm{SCH}_{3}\right), 111.9\left(\mathrm{C}_{10}\right), 116.9\left(\mathrm{C}_{8}\right), 119.5\left(\mathrm{C}_{6}\right), 124.2\left(\mathrm{C}_{5}\right)$, $128.8\left(\mathrm{C}_{11}{ }^{*}\right), 129.3\left(\mathrm{C}_{9} *\right), 157.8\left(\mathrm{C}_{4}\right)$. Anal. Calcd for $\mathrm{C}_{14} \mathrm{H}_{10} \mathrm{~N}_{4} \mathrm{~S}: \mathrm{C}, 63.14$; H, 3.78; N, 21.04. Found: C, 63.25; H, 3.99; N, 21.13.

10-Methylpyrido $\left[\mathbf{1}^{\prime}, \mathbf{2}^{\prime}: \mathbf{1 , 2}\right]$ imidazo[4,5-h]quinazoline (26) This compound was obtained according to the procedure used for $\mathbf{2 3}$ (reaction time, $2 \mathrm{~h}$ ); recrystallization in ether. Yield: $7 \%$. mp: $207-209^{\circ} \mathrm{C}$. MS $\mathrm{m} / \mathrm{z}: 236$ (8), 235 (29), 234 (100), 233 (58). ${ }^{1} \mathrm{H}-\mathrm{NMR}\left(\mathrm{CDCl}_{3}, 100 \mathrm{MHz}\right) \delta: 2.56$ $\left(\mathrm{s}, \mathrm{CH}_{3}\right), 7.48\left(\mathrm{~d}, 1 \mathrm{H}, J_{8-9}=8.8 \mathrm{~Hz}, \mathrm{H}_{9}\right), 7.84\left(\mathrm{~d}, 1 \mathrm{H}, \mathrm{H}_{8}\right), 7.86(\mathrm{~d}, 1 \mathrm{H}$, $\left.J_{5-6}=8.8 \mathrm{~Hz}, \mathrm{H}_{5}\right), 8.10\left(\mathrm{~d}, 1 \mathrm{H}, \mathrm{H}_{6}\right), 9.42\left(\mathrm{~s}, 2 \mathrm{H}, \mathrm{H}_{2}, \mathrm{H}_{4}\right), 9.86\left(\mathrm{~s}, 1 \mathrm{H}, \mathrm{H}_{11}\right)$. ${ }^{13} \mathrm{C}-\mathrm{NMR}\left(\mathrm{CDCl}_{3}, 25 \mathrm{MHz}\right) \delta_{\mathrm{CH}}: 18.5\left(\mathrm{CH}_{3}\right), 116.9\left(\mathrm{C}_{8}\right), 122.5\left(\mathrm{C}_{6}\right), 124.3$ $\left(\mathrm{C}_{5}\right), 127.6\left(\mathrm{C}_{11}{ }^{*}\right), 133.0\left(\mathrm{C}_{9}{ }^{*}\right), 155.1\left(\mathrm{C}_{4}\right), 158.4\left(\mathrm{C}_{2}\right)$. Anal. Calcd for $\mathrm{C}_{14} \mathrm{H}_{10} \mathrm{~N}_{4}: \mathrm{C}, 71.78 ; \mathrm{H}, 4.30 ; \mathrm{N}, 23.92$. Found: $\mathrm{C}, 71.75 ; \mathrm{H}, 4.26 ; \mathrm{N}, 23.99$.

10-Chloropyrido $\left[\mathbf{1}^{\prime}, \mathbf{2}^{\prime}: \mathbf{1}, 2\right]$ imidazo[4,5-h]quinazoline (27) This compound was obtained according to the procedure used for $\mathbf{2 3}$ (reaction time, $10 \mathrm{~h}$ ); chromatography: silica gel with $\mathrm{CH}_{2} \mathrm{Cl}_{2} / \mathrm{CH}_{3} \mathrm{OH}(98 / 2)$. Yield: $52 \%$. mp: 216-218 ${ }^{\circ} \mathrm{C}$. MS $m / z 254$ (25), 221 (25), 167 (57), 149 (80). ${ }^{1} \mathrm{H}-\mathrm{NMR}$ $\left(\mathrm{CDCl}_{3}, 100 \mathrm{MHz}\right) \delta: 7.59\left(\mathrm{~d}, 1 \mathrm{H}, \mathrm{H}_{9}, J_{8-9}=9.1 \mathrm{~Hz}\right), 7.80-8.10(\mathrm{~m}, 3 \mathrm{H}$, $\left.\mathrm{H}_{5}, \mathrm{H}_{6}, \mathrm{H}_{8}\right), 9.44\left(\mathrm{~s}, 2 \mathrm{H}, \mathrm{H}_{2}, \mathrm{H}_{4}\right), 10.09\left(\mathrm{~s}, 1 \mathrm{H}, \mathrm{H}_{11}\right) \cdot{ }^{13} \mathrm{C}-\mathrm{NMR}\left(\mathrm{CDCl}_{3}\right.$, $25 \mathrm{MHz}) \delta_{\mathrm{CH}}: 117.3\left(\mathrm{C}_{10}\right), 126.1\left(\mathrm{C}_{6}\right), 128.2\left(\mathrm{C}_{5}\right), 128.9\left(\mathrm{C}_{9}{ }^{*}\right), 130.9\left(\mathrm{C}_{11}{ }^{*}\right)$, $153.6\left(\mathrm{C}_{2}\right), 157.0\left(\mathrm{C}_{4}\right)$. Anal. Calcd for $\mathrm{C}_{13} \mathrm{H}_{7} \mathrm{~N}_{41}: \mathrm{C}, 61.31 ; \mathrm{H}, 2.77 ; \mathrm{N}$, 22.00. Found: C, $61.45 ; \mathrm{H}, 2.58 ; \mathrm{N}, 21.91$

Biological Assay Doxorubicin hydrochloride (Pharmacia, St Quentin en Yvelines, France); RPMI 1640 medium and fetal calf serum (Polylabo, Paris, France) were used in this study. All other reagents were of analytical grade and were obtained from commercial sources.

Cells and Cultures: The human promyelocytic leukaemia cell line, HL60 was obtained from the American type culture collection (Rockville, MD, U.S.A.). The human ovarian carcinoma were generously given by $\mathrm{Dr}$ P. Canal (C. R. L. C. Val d'Aurelle). The doxorubicin resistant sublines HL60R and A2780R were established by the continuous spasm of cells to gradually increasing concentrations of daunorubicin and doxorubicine, respectively, and were maintained in medium supplemented with daunorubicin and doxorubicin at $0.1 \mu \mathrm{g} / \mathrm{ml}$, respectively. The MDR phenotype expression of the HL60R and A2780R cell lines was assessed by an immunohistochemistry method, using the two P-glycoprotein-specific murine monoclonal antibodies C219 (Cantocor, Malvern, PA, U.S.A.) and JSB1 (Tebu, le Perray en 
Yvelines, France). Cultures were grown in RPMI 1640 medium supplemented with $10 \%$ fetal calf serum, antibiotics and glutamine at $37^{\circ} \mathrm{C}$ in a humidified atmosphere containing $5 \% \mathrm{CO}_{2}$.

Cytotoxicity assays: In all experiments, parental sensitive and resistant HL60 and A2780 cells were seeded at a final density of 6000 cells/well in 96 well microtiter plates and were treated with drugs (doxorubicin and compounds 16, 21, 23, 24). Ten dilutions were used for each drug. After $96 \mathrm{~h}$ of incubation, $20 \mu \mathrm{l}$ of MTT solution in PBS $(5 \mathrm{mg} / \mathrm{ml})$ was added to each well and the wells were then exposed to $37^{\circ} \mathrm{C}$ for $4 \mathrm{~h}$. This colorimetric assay is based on the ability of live and metabolically unimpaired tumor-cell targets to reduce MTT to a blue formazan product. ${ }^{8)}$ Then, $100 \mu \mathrm{l}$ of a mixture of isopropanol and 1м hydrochloride acid $(96: 4, \mathrm{v} / \mathrm{v})$ was added to each well. After a vigorous shaking, the absorbance was measured on a microculture plate reader (Dynatech MR5000, France) at $570 \mathrm{~nm}$. For each assay, at least four experiments were performed in triplicate. The resistance factor (RF) was calculated from the ratio between the $\mathrm{IC}_{50} \%$ growth-inhibitory concentrations $\left(\mathrm{IC}_{50}\right.$ values) recorded from HL60 R, A2780 R and HL60, A2780 cells, respectively, for all drugs tested (doxorubicin; compounds 16, 21, 23, 24).

Acnowledgements We gratefully acknowledge financial support from the Ligue Nationale de Lutte contre le Cancer (Comité de l'Hérault).

\section{References and Notes}

1) Blache Y., Gueiffier A., Chavignon O., Teulade J.-C., Milhavet J.-C., Viols H., Chapat J.-P., Dauphin G., J. Heterocyclic Chem., 31, 161166 (1994); Chavignon O., Teulade J.-C., Roche D., Madesclaire M., Blache Y., Gueiffier A., Chabard J.-L., Dauphin G., J. Org. Chem., 59, 6413-6418 (1994); Gueiffier A., Viols H., Blache Y., Chavignon O.,
Teulade J.-C., Chapat J.-P., Fauvelle F., Grassy G., Dauphin G., J. Heterocyclic Chem., 34, 765-771 (1997).

2) Blache Y., Sinibaldi-Troin M. E., Voldoire A., Chavignon O., Gramain J. C., Teulade J. C., Chapat J. P., J. Org. Chem., 62, 8553-8556 (1997); Blache Y., Chavignon O., Sinibaldi-Troin M. E., Gueiffier A., Teulade J. C., Troin Y., Gramain J. C., Heterocycles, 38, 1241-1246 (1994).

3) Badaway E., Kappe T., Eur. J. Med. Chem., 30, 327-332 (1995).

4) Dupuy M., Pinguet F., Chavignon O., Teulade J. C., Chapat J. P, Blache Y., Heterocyclic Chem., 7, 23-28 (2001).

5) Dupuy M., Pinguet F., Blache Y., Chavignon O., Teulade J. C., Chapat J. P., Chem. Pharm. Bull., 46, 1820-1823 (1998).

6) Chevillard S., Vielh P., Oncologia, 5, 5-13 (1993).

7) Grimble G. W., "The Alkaloids," Vol. 36, Academic Press, Inc., Vol. 36, 135 1989, p. 135-170.

8) Riou J. F., Fosse P., Nguyen C. H., Kragh-Larsen A., Bissery M. C., Grondard L., Saucier J. M., Bisagni E., Lavelle F., Cancer Res., 53, 5987-5993 (1993).

9) Van Gijn R., Huinick T. B., Rodenhuis S., Vermorken J. B., Van Telligen O., Rosing H., Van Warmerdam L. J., Beijnen J. H., Anticancer Drugs, 10, 17-23 (1999); Abigerges J. P., Chabot C. G., Bruno R., Bisser M. C., Bayssas M., Klinkl-Alakl M., Clavel M., Catimel G., ibid., 7, 166-174 (1996).

10) Chauffert B., Correia M., Sergent C., Bull. Cancer, 86, 97-103 (1999); Schinkel A. H., Semin. Cancer Biol., 8, 161-170 (1997).

11) Blache Y., Gueiffier A., Chavignon O., Teulade J. C., Chapat J. P., Heterocyclic Com., 2, 331-337 (1996).

12) Bowen M. C., Katenellenbogen J. A., J. Org. Chem., 62, 7650-7657 (1997). 